\title{
A Systematic Review and Meta-Analysis of Nonpharmacological Interventions for Moderate to Severe Dementia
}

\author{
Riyoung $\mathrm{Na}^{1}$, Ji-hye Yang1, Yusung Yeom ${ }^{1}$, You Joung Kim¹, \\ Seonjeong Byun ${ }^{1,2}$, Kiwon Kim ${ }^{1,2}$, and Ki Woong Kim ${ }^{1,2,3,4} \bowtie$ \\ ${ }^{1}$ National Institute of Dementia, Seongnam, Republic of Korea \\ ${ }^{2}$ Department of Neuropsychiatry, Seoul National University Bundang Hospital, Seongnam, Republic of Korea \\ ${ }^{3}$ Department of Psychiatry, Seoul National University College of Medicine, Seoul, Republic of Korea \\ ${ }^{4}$ Department of Brain and Cognitive Science, Seoul National University College of Natural Sciences, Seoul, Republic of Korea
}

Objective Due to limited efficacy of medications, non-pharmacological interventions (NPI) are frequently co-administered to people with moderate to severe dementia (PWMSD). This systematic review and meta-analysis investigated the effects of NPI on activities of daily living (ADL), behavioral and psychological symptoms of dementia (BPSD), and cognition and quality of life (QoL) of PWMSD.

Methods A literature search was conducted in the following databases: Cochrane CENTRAL, EMBASE, Medline, CIHNAL, PsycINFO, KoreaMED, KMbase, and KISS. We conducted a meta-analysis on randomized controlled trials and used the generic inverse variance method with a fixed-effects model to calculate the standardized mean difference (SMD). The protocol had been registered (CRD42017 058020).

Results Ten randomized controlled trials met the inclusion criteria of the current meta-analysis. NPI were effective in improving ADL $[\mathrm{SMD}=0.28,95 \%$ confidence interval $(\mathrm{CI})=0.11-0.45]$ and reducing depression $(\mathrm{SMD}=-0.44,95 \% \mathrm{CI}=-0.70--0.19)$. However, NPI were not effective in reducing agitation, anxiety, or overall, or improving cognitive function. In a subgroup analysis, music therapy was effective in reducing overall BPSD (SMD=-0.52, 95\% CI=-0.90- -0.13 ).

Conclusion Albeit the number of studies was limited, NPI improved ADL and depression in PWMSD.

Psychiatry Investig 2019;16(5):325-335

Key Words Dementia, Nonpharmacological intervention, Activities of daily living, Depression, Behavioral and psychological symptoms of dementia.

\section{INTRODUCTION}

People with dementia (PWD) experience gradual but progressive loss of cognition, and more than half of them suffer from behavioral and psychological symptoms. ${ }^{1}$ However, the efficacy of cholinesterase inhibitors and N-methyl-d-aspartate (NMDA) receptor antagonists is limited, particularly in cases of moderate to severe dementia. ${ }^{2-5}$ As such, new anti-dementia drugs in clinical trials are targeting prodromal or earlystage dementia. ${ }^{6}$ Antipsychotics, which are commonly pre-

Received: November 27, 2018 Accepted: February 11, 2019

$\bowtie$ Correspondence: Ki Woong Kim, MD, PhD

Department of Neuropsychiatry, Seoul National University Bundang Hospital, 82 Gumi-ro 173beong-gil, Bundang-gu, Seongnam 13620, Republic of Korea Tel: +82-31-787-1821, Fax: +82-31-628-6848, E-mail: kwkimmd@snu.ac.kr

(c) This is an Open Access article distributed under the terms of the Creative Commons Attribution Non-Commercial License (https://creativecommons.org/licenses/by$\mathrm{nc} / 4.0$ ) which permits unrestricted non-commercial use, distribution, and reproduction in any medium, provided the original work is properly cited. scribed for behavioral and psychological symptoms of dementia (BPSD), are associated with serious adverse effects, including pneumonia, cardiovascular events, stroke, fractures, and kidney failure. ${ }^{7,8}$ Above all, pharmacological interventions cannot fulfill the needs of PWD and their caregivers, including relief of pain and discomfort, the need for social contact, and alleviation of boredom. ${ }^{9}$ For these reasons, a combination of pharmacological and nonpharmacological interventions (NPI) is strongly recommended for PWD. ${ }^{10-13}$

Recent systematic reviews have identified the effects of various NPI on cognitive decline, ${ }^{14-16} \mathrm{BPSD},{ }^{12-17}$ activities of daily living (ADL) $)^{14,16,18}$ and quality of life (QoL) ${ }^{14}$ of PWD. However, most analyses in previous systematic reviews did not take into account the severity of dementia. ${ }^{17,19-23}$ Although there have been several systematic reviews focused on the effects of NPI in people with moderate to severe dementia (PWMSD), they did not conduct meta-analyses ${ }^{24,25}$ or con- 
ducted a meta-analysis on the effects of NPI in PWMSD as a subgroup analysis only. ${ }^{15,26,27}$ In this study, we conducted a systematic review and meta-analysis to evaluate the efficacy of NPI on the cognitive function, BPSD, and ADL of PWMSD.

\section{METHODS}

We conducted a systematic review in accordance with the Preferred Reporting Items for Systematic Reviews and MetaAnalyses (PRISMA) Statement ${ }^{28}$ and the Cochrane Handbook for Systematic Reviews of Interventions. ${ }^{29}$ The study protocol was previously published, ${ }^{30}$ and it is registered with the International Prospective Register of Systematic Reviews (PROSPERO, CRD42017058020).

\section{Search strategy}

We identified the studies that investigated the efficacy of NPI in PWMSD through bibliographic databases such as the Cochrane Central Register of Controlled Trials (CENTRAL), EBSCO-EMBASE, Proquest-Medline, ProQuest-PsycINFO, EBSCO-CINAHL, KoreaMED, KMbase, and Koreanstudies Information Service System. We also searched the reference lists of previous systematic reviews on the efficacy of NPI in PWD to extract relevant papers.

The search strategy combined several Medical Subject Headings or Emtree terms of population and intervention to identify relevant studies. The search terms were adapted using truncation or Boolean operators with database-specific terms. The population included PWMSD who were identified using the following search terms: [Dementia; Alzheimer Disease; Dementia, Vascular; Lewy Body Disease; Frontotemporal Dementia; Hydrocephalus, Normal Pressure; Huntington Disease; Neurodegenerative Disease; alcohol related dementia; mental disorder*; Parkinson's disease dementia; moderate; severe; moderate to severe; advanced; profound]. Interventions included any NPI identified using the following search terms: [Psychotherapy; Cognitive Therapy; Behavior Therapy; Aromatherapy; Massage; Music Therapy; Animal Assisted Therapy; Exercise; Art Therapy; Horticultural Therapy; Occupational Therapy; Telerehabilitation; Therapy, Computer-Assisted; Dance Therapy; Play Therapy; Reality Therapy; Recreation Therapy; non pharmacological; non drug; light therap*; snoezelen; multimodality therap*; multisensory; doll therapy; robot therapy; cognitive training]. The literature searches were conducted April 18, 2017.

\section{Study selection and inclusion criteria}

We exported the search results to EndNote ${ }^{\mathrm{TM}} \mathrm{X} 8$ (Clarivate Analytics, USA), and three reviewers (RN, JY, and YY) independently assessed the results for inclusion by title, abstract and full text. Other reviewers (YJK, SB, KWK, and KK) resolved any discrepancies among the initial three reviewers regarding the selection of studies.

The systematic review included studies involving people with any type of dementia according to the standardized diagnostic criteria in the Diagnostic and Statistical Manual of Mental Disorders; ${ }^{31-33}$ the International Classification of Diseases, Tenth Revision; ${ }^{34}$ the National Institute of Neurological and Communicative Disorders and Stroke and the Alzheimer's Disease and Related Disorders Association; ;5,36 or other recommended diagnostic criteria. To be considered moderate to severe, dementia had to meet one of the following criteria: a Clinical Dementia Rating score ${ }^{37}$ of 2 or more, a Global Deterioration Scale ${ }^{38}$ score of 5 or more, a Functional Assessment Staging ${ }^{39}$ score of 5 or more, or a Mini-Mental State Examination $\left(\mathrm{MMSE}^{40}\right)$ score of 20 or less. If the range of severity score was not reported, the determination of severity was based on the mean and standard deviations score of the MMSE. The intervention criteria had no restrictions regarding category of NPI of practice guidelines. ${ }^{41}$

We included randomized controlled trials (RCTs), quasiRCTs, non-RCTs, cross-sectional studies, interrupted time series, and before-after studies that used the Study Design Algorithm for Medical Literature on Intervention. ${ }^{42}$ We limited the publication languages to English and Korean, but did not limit the geography, time of the study, or publication year.

\section{Outcomes}

Primary outcomes included ADL and BPSD; secondary outcomes included cognitive function and QoL. These outcomes were evaluated using standardized scales for validity.

\section{Data extraction}

Three reviewers (RN, JY, and YY) independently extracted relevant data from articles using a standardized form. The form included details of the participants (e.g., number of patients, characteristics, demographics, and dementia severity), interventions (e.g., type, provider, period of intervention, and setting), and results (e.g., outcome, measurement scales, and result data). Other reviewers (YJK, SB, KWK, and KK) resolved any disagreements among the initial reviewers.

\section{Assessment of methodological quality}

Three reviewers (RN, JY, and YY) independently assessed the studies' methodological quality and risk of bias (RoB) according to standardized tools and criteria. All reviewers discussed the quality of each study if the initial reviewers disagreed. We evaluated the RoB of RCTs using the RoB scale, ${ }^{43}$ and the quality of evidence for outcomes using the Grading of Recommendations Assessment, Development and Evaluation 
$\left(\mathrm{GRADE}^{44}\right)$. All reviewers conferred regarding the quality of the evidence according to the GRADE guidelines. ${ }^{44}$

\section{Data analysis}

We conducted meta-analyses on RCTs that reported the efficacy of NPI using valid scales, and attempted to contact the authors of studies with missing data to obtain the relevant information. We synthesized the published data and the data obtained from the authors. We used the generic inverse variance method with a fixed-effects model to calculate the standardized mean difference (SMD), as the included studies assessed the same outcomes but measured them using a variety of scales. ${ }^{29}$ We performed subgroup analysis to assess whether differences in treatment affected outcomes. We utilized the fixed-effects model for the following reasons: 1) the population groups of included studies were similar, and 2) there was a limited number of studies to synthesize. The meta-analysis was performed using Review Manager, version 5.3 (Copen- hagen: The Nordic Cochrane Centre, The Cochrane Collaboration). We assessed heterogeneity using chi-squared and Isquared tests. We did not evaluate publication biases due to an insufficient number of studies. ${ }^{29}$

\section{RESULTS}

\section{Study identification and selection}

We identified 9,219 references in the selected databases. After removing the duplicates and clearly irrelevant articles, we retrieved 1,183 full-text records. Of these, we excluded another irrelevant 594 references and added 11 additional references identified by the authors, leaving 600 full-text references to be assessed for eligibility. Of these, we excluded 577 studies, as they did not meet the inclusion and exclusion criteria. Finally, there were 23 studies eligible for inclusion. Among them, only $10 \mathrm{RCTs}^{45-54}$ and one Cluster-RCT ${ }^{55}$ met the criteria for meta-analysis (Figure 1).

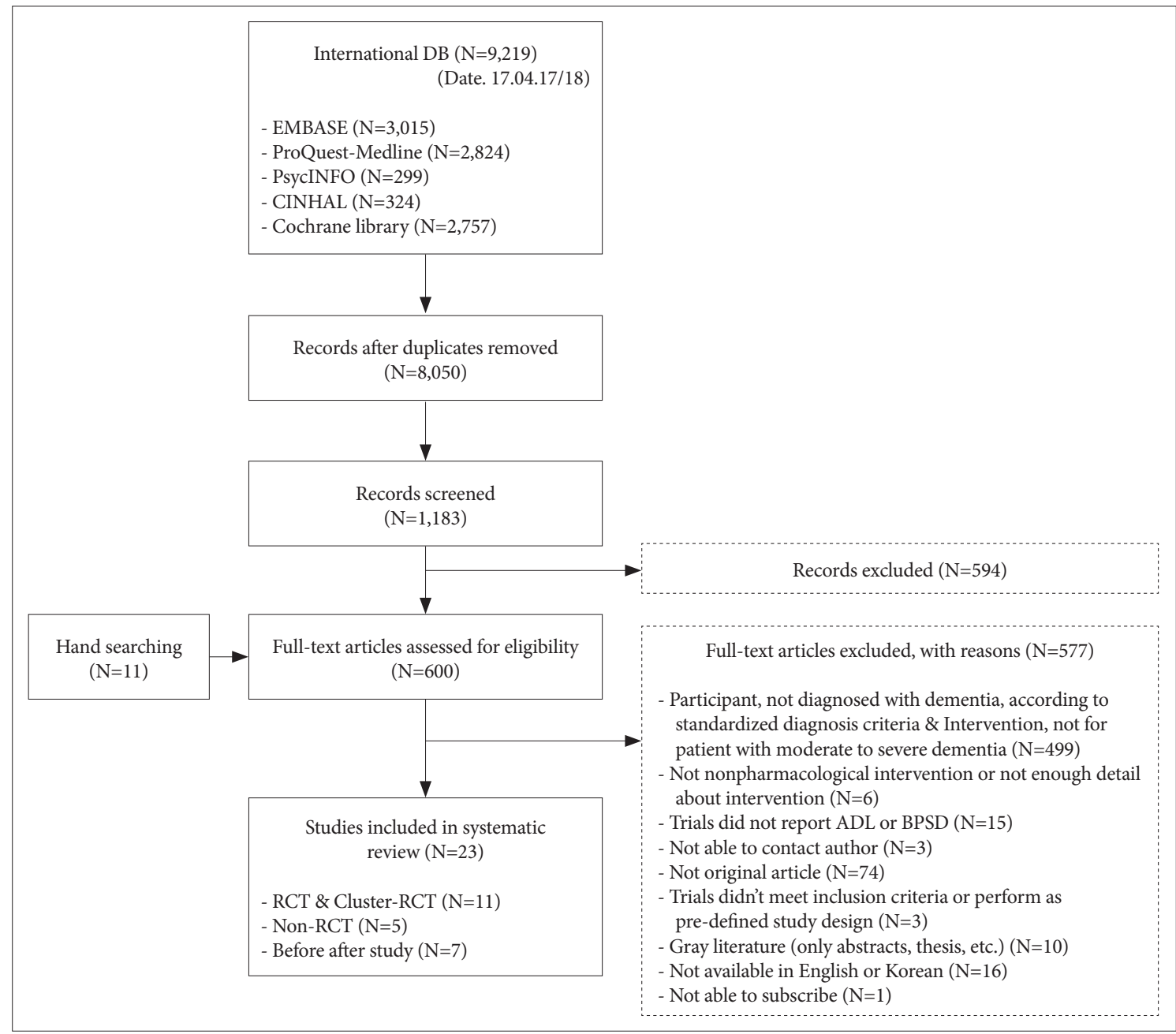

Figure 1. Preferred Reporting Items for Systematic Reviews and Meta-Analyses (PRISMA) flow. 


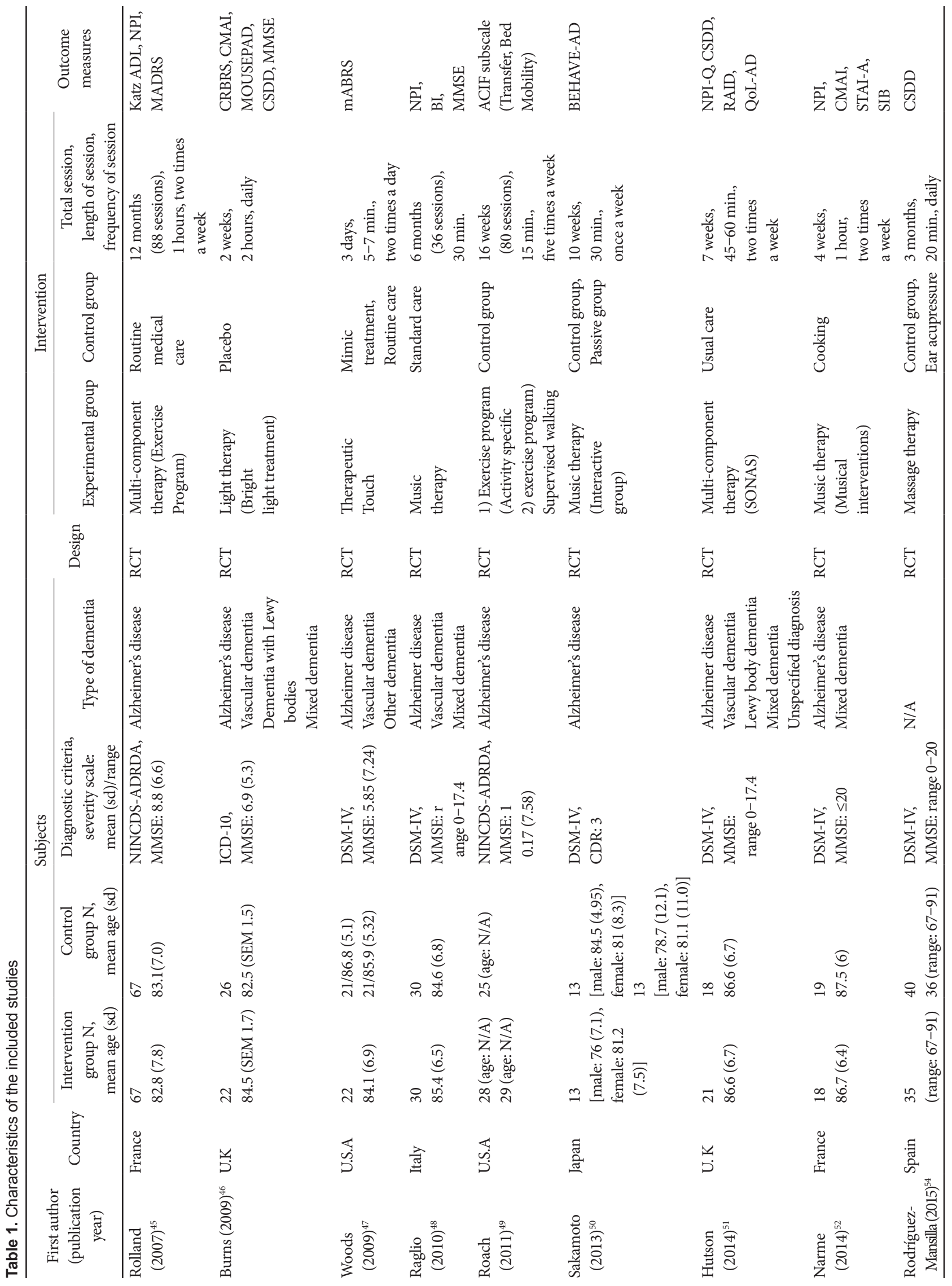




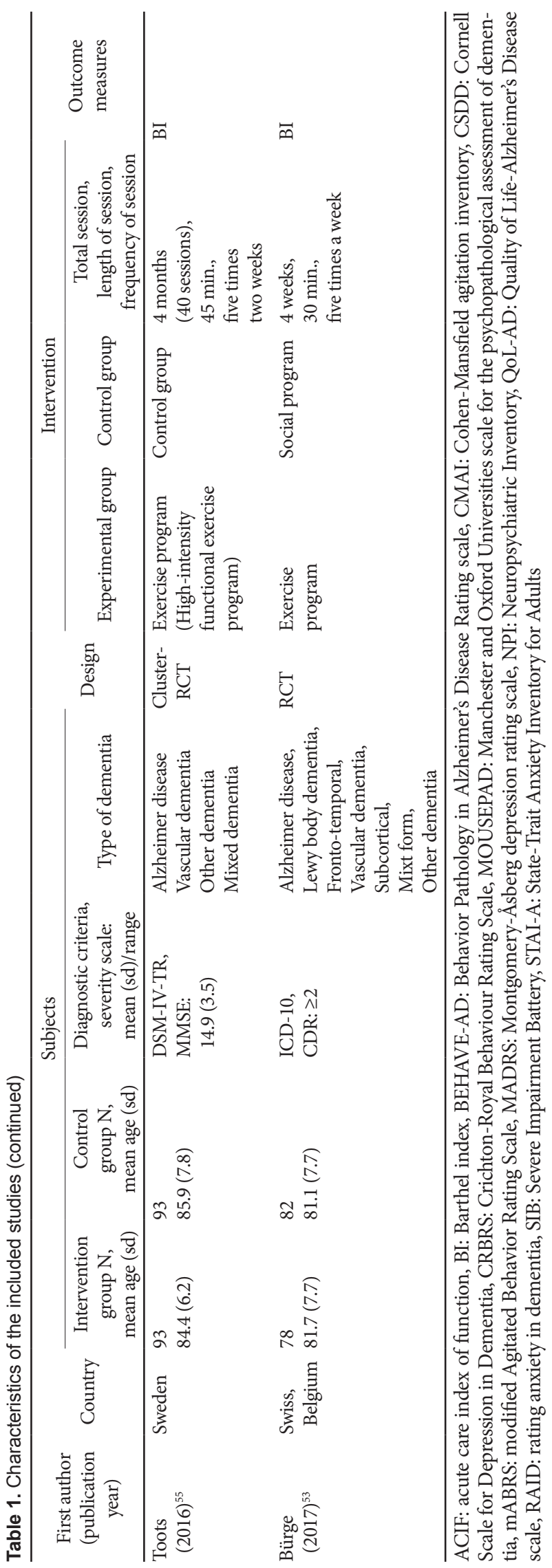

\section{Characteristics of included studies}

Characteristics of the ten RCTs ${ }^{45-54}$ and one Cluster-RCT ${ }^{55}$ are summarized in Table 1. These eleven studies included 960 PWMSD (intervention group: 456; control group: 504). Ten studies included patients with Alzheimer's disease; six studies included patients with vascular dementia or other types of dementia such as Lewy body dementia or frontal dementia. All interventions provided to PWMSD in these studies utilized stimulation-oriented approaches. ${ }^{41}$ Three interventions used music and exercise therapies, two used multi-component therapies, two used massage (including therapeutic touch) therapies, and one used light therapy.

The validity of the instruments employed in the studies to evaluate primary and secondary outcomes depended on the study references: Katz ADL, ${ }^{56}$ Barthel Index,${ }^{57,58}$ and Crichton-Royal Behavioural Rating Scale ${ }^{59}$ for ADL; Neuropsychiatric Inventory, ${ }^{60,61}$ Neuropsychiatric Inventory Questionnaire, ${ }^{62}$ Behavior Pathology in Alzheimer's Disease Rating Scale ${ }^{63}$ and Manchester and Oxford Universities Scale for the Psychopathological Assessment of Dementia ${ }^{64}$ for overall BPSD; Cornell Scale for Depression in Dementia, ${ }^{65}$ and Montgomery-Åsberg Depression Rating Scale $e^{66,67}$ for depression; Cohen-Mansfield Agitation Inventory, ${ }^{68}$ and modified Agitated Behavior Rating Scale ${ }^{69}$ for agitation; Rating Anxiety in Dementia ${ }^{70}$ and State-Trait Anxiety Inventory for Adults ${ }^{71}$ for anxiety; and MMSE and Severe Impairment Battery ${ }^{72}$ for global cognitive function.

\section{Effects of NPI on ADL}

Six studies reported the effect of NPI on ADL: multi-component therapies in two studies, ${ }^{45,53}$ exercise therapies in two studies ${ }^{49,55}$ light therapy in one study, ${ }^{46}$ and music therapy in one study ${ }^{48}$ We excluded one study ${ }^{49}$ from the meta-analysis because it reported only subscale results. NPI had a beneficial effect on $\mathrm{ADL}$ when compared to the control $[\mathrm{SMD}=0.28$, $95 \%$ confidence interval $(\mathrm{CI})=0.11-0.45, \mathrm{p}=0.001]$. There was no heterogeneity across the trials $\left(\mathrm{I}^{2}=0 \%\right)$ (Figure 2 ).

\section{Effects of NPI on BPSD}

Six studies reported the effect of NPI on the overall BPSD: multi-component therapies in two studies ${ }^{45,51}$ music therapies in three studies, ${ }^{48,50,52}$ and light therapy in one study. ${ }^{46} \mathrm{NPI}$ did not show a beneficial effect on the overall BPSD when compared to the control (SMD $=-0.16,95 \% \mathrm{CI}=-0.39-0.06, \mathrm{p}=0.16)$. The heterogeneity between studies was considerable $\left(\mathrm{I}^{2}=81 \%\right){ }^{29}$ In the subgroup analysis, music therapy showed a beneficial effect on the overall BPSD compared to the control (SMD= $-0.52,95 \% \mathrm{CI}=-0.90--0.13, \mathrm{p}=0.008)$, but there was high heterogeneity between studies $\left(\mathrm{I}^{2}=90 \%\right)^{29}$ (Figure 3 ).

There were four studies that reported the effect of NPI on 


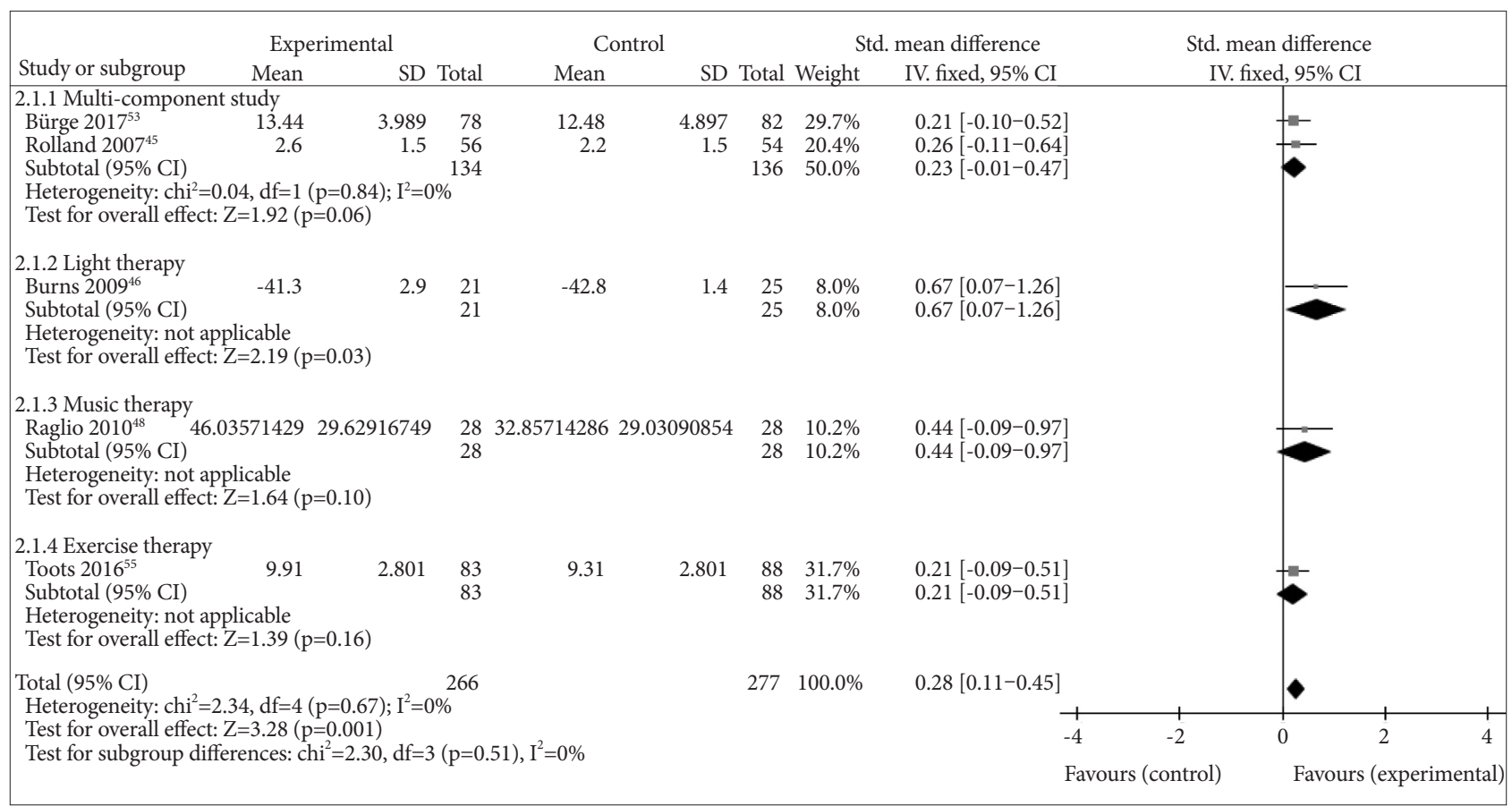

Figure 2. Meta-analysis on activity of daily living.

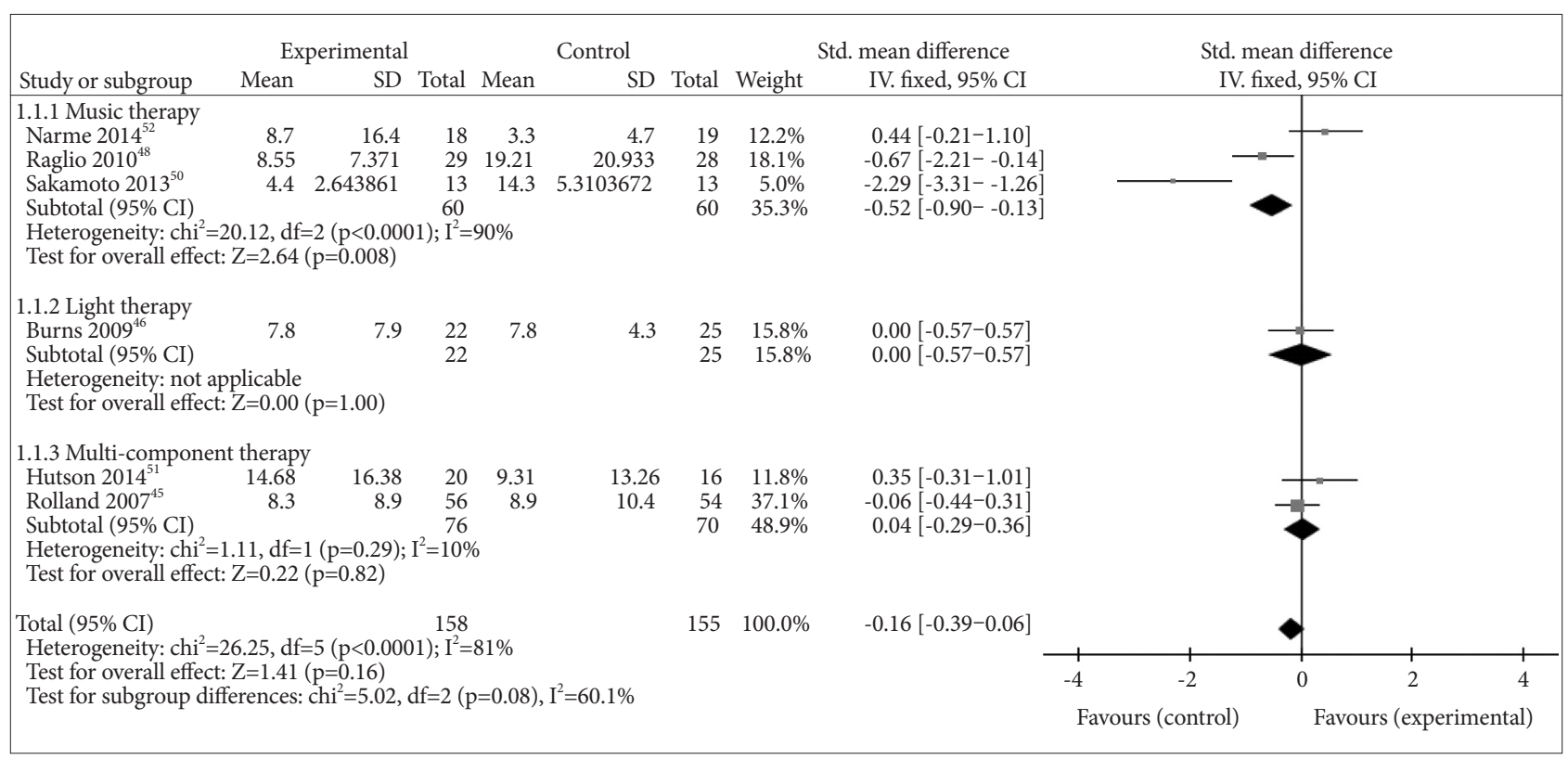

Figure 3. Meta-analysis on overall Behavioral and Psychological Symptoms of Dementia (BPSD).

depression separately: multi-component therapies in two studies, ${ }^{45}, 51$ light therapy in one study, ${ }^{46}$ and massage therapy in one study ${ }^{54}$ Compared to the control, NPI had a positive effect on depression $(\mathrm{SMD}=-0.44,95 \% \mathrm{CI}=-0.70--0.19, \mathrm{p}=0.0007)$, but the heterogeneity between studies was substantial $\left(\mathrm{I}^{2}=\right.$ 93\%) (Figure 4).

Three studies reported the effect of NPI on agitation separately: ${ }^{46,47,52}$ light therapy in one study, ${ }^{46}$ music therapy in one study, ${ }^{52}$ and massage therapy in one study ${ }^{47}$ NPI did not have a beneficial effect on agitation $(\mathrm{SMD}=0.14,95 \% \mathrm{CI}=-0.21-$ $0.49, \mathrm{p}=0.43$ ), and there was no heterogeneity between studies $\left(\mathrm{I}^{2}=0 \%\right)$ (Figure 5$)$.

We identified two studies that reported the effect of NPI on anxiety separately: ${ }^{51,52}$ multi-component therapy in one study ${ }^{51}$ and music therapy in one study. ${ }^{52}$ NPI did not have a beneficial effect on anxiety $(\mathrm{SMD}=0.01,95 \% \mathrm{CI}=-0.45-0.48, \mathrm{p}=0.95)$, 


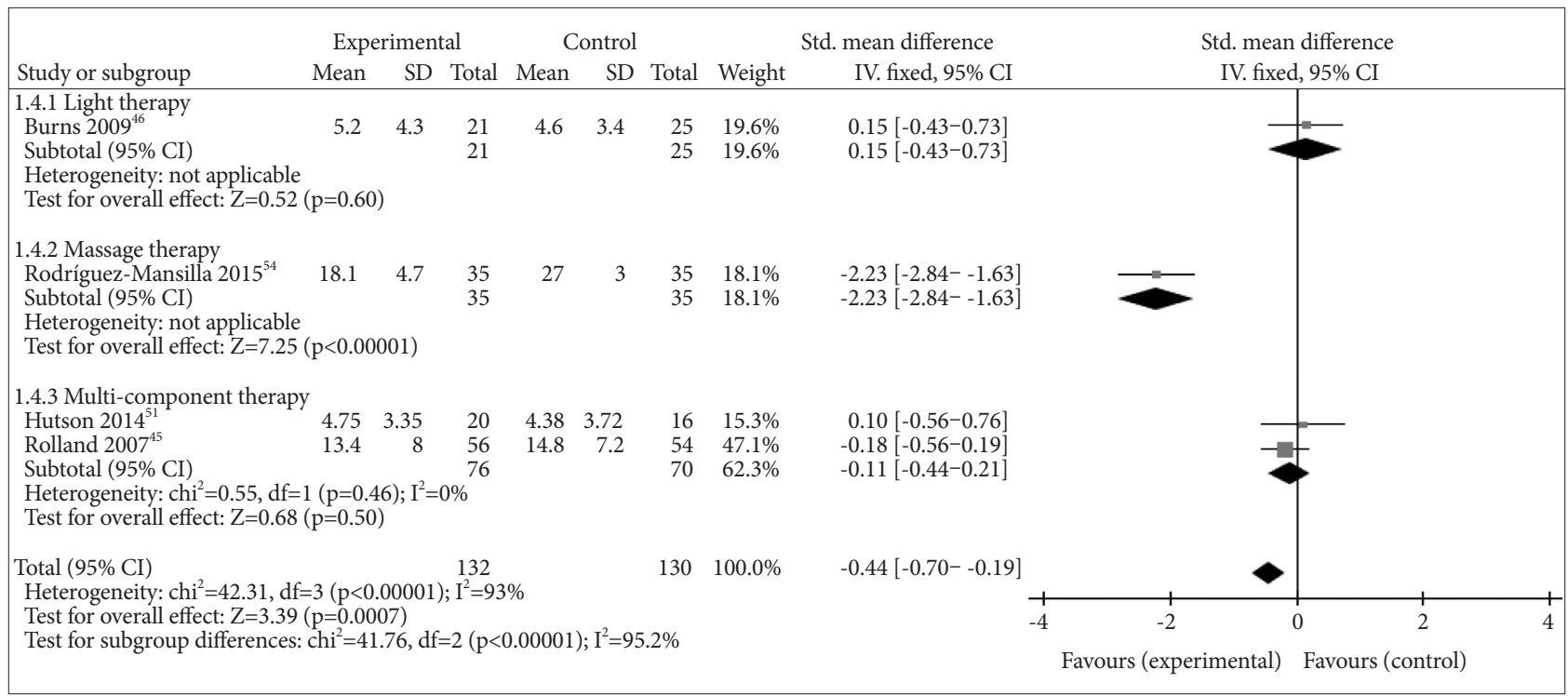

Figure 4. Meta-analysis on depression.

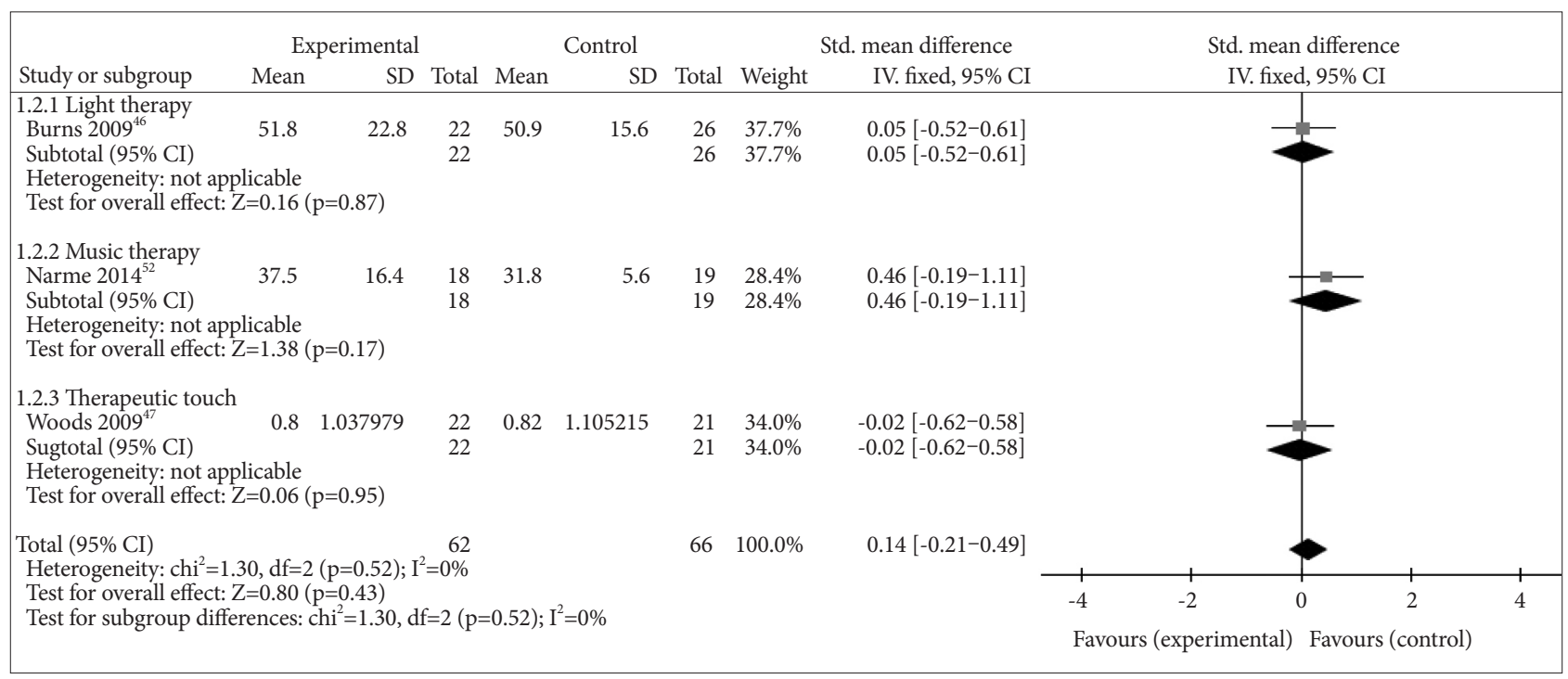

Figure 5. Meta-analysis on agitation.

and there was no heterogeneity between studies $\left(\mathrm{I}^{2}=0 \%\right)$ (Figure 6).

\section{Effects of NPI on cognitive function}

Three studies evaluated the effect of NPI on cognitive function: music therapy in two studies ${ }^{48,52}$ and light therapy in one study. ${ }^{46} \mathrm{NPI}$ did not have a beneficial effect on cognitive function $(\mathrm{SMD}=0.30,95 \% \mathrm{CI}=-0.04-0.64, \mathrm{p}=0.08)$, and there was no heterogeneity between studies (Figure 7).

\section{Effects of NPI on QoL}

Since there was only one study that reported the effect of multi-component study on QoL, we did not conduct a meta- analysis on the effect of NPI on QoL. ${ }^{51}$ In that study, NPI were not beneficial to QoL when compared to the control (SMD= $-0.65,95 \% \mathrm{CI}=-4.79-3.49, \mathrm{p}=0.76)$.

\section{DISCUSSION}

Results of the current study are summarized in Table 2. NPI were shown to improve ADL of PWMSD. Although the current meta-analysis encompassed a wide range of NPI such as exercise therapy, light therapy, music therapy, and multi-component therapy, there was no heterogeneity between studies and subgroups. Light therapy had a strong beneficial effect on ADL when compared to the control. Although the efficacies 


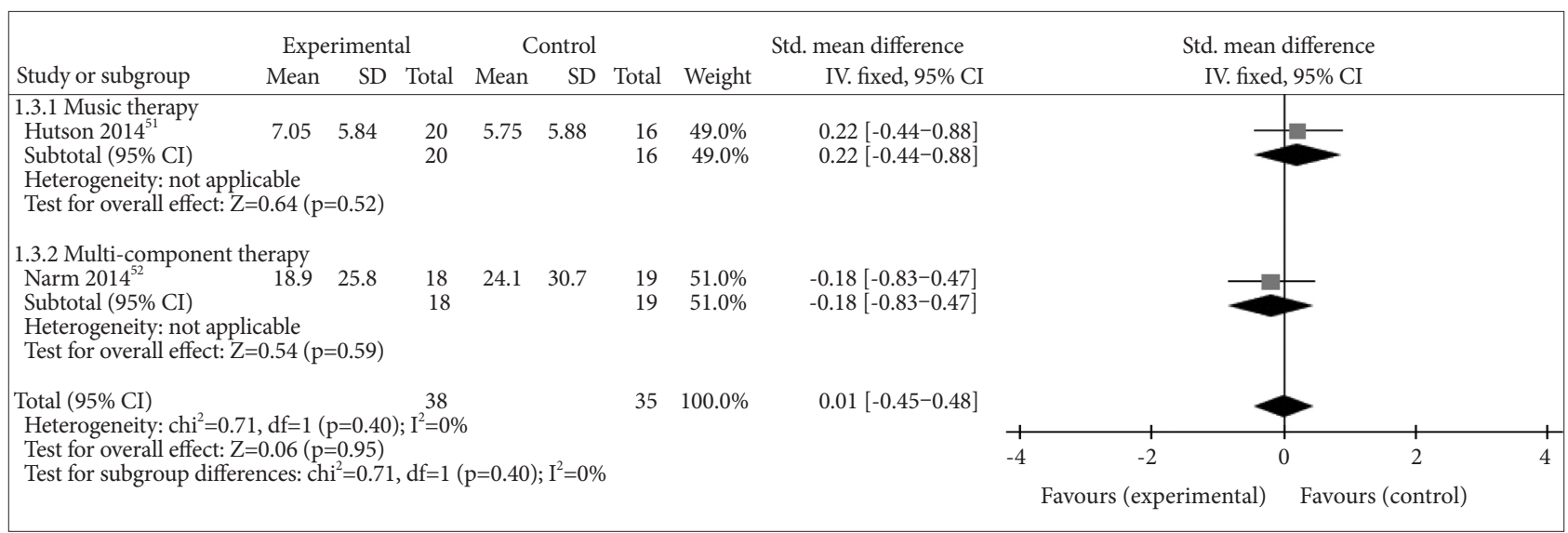

Figure 6. Meta-analysis on anxiety.

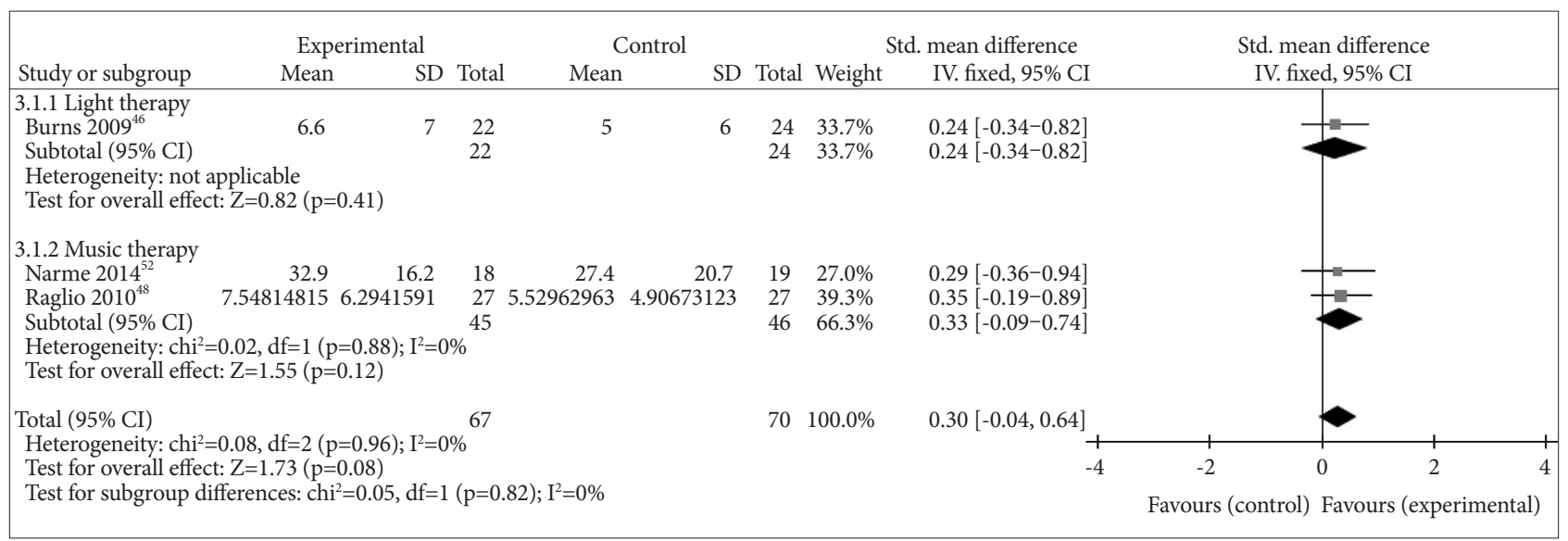

Figure 7. Meta-analysis on cognitive function.

Table 2. Summary of finding table

\begin{tabular}{llcc}
\hline \multicolumn{1}{c}{ Outcomes } & \multicolumn{1}{c}{$\begin{array}{c}\text { Anticipated absolute effects (95\% CI) } \\
\text { risk with non-pharmacological interventions }\end{array}$} & $\begin{array}{c}\text { No of participants } \\
\text { (studies) }\end{array}$ & $\begin{array}{c}\text { Certainty of the evidence } \\
\text { (GRADE) }\end{array}$ \\
\hline ADL & SMD 0.28 SD higher (0.11 higher to 0.45 higher) & 543 (5 RCTs) & MODERATE \\
Overall BPSD & SMD 0.16 lower (0.39 lower to 0.06 higher) & 313 (6 RCTs) & VERY LOW \\
Depression & SMD 0.44 lower (0.7 lower to 0.19 lower) & 262 (4 RCTs) & VERY LOW \\
Agitation & SMD 0.14 higher (0.21 lower to 0.49 higher) & 128 (3RCTs) & LOW \\
Anxiety & SMD 0.01 higher (0.45 lower to 0.48 higher) & 73 (2RCTs) & MODERATE \\
Cognitive function & SMD 0.3 higher (0.04 lower to 0.64 higher) & 137 (3 RCTs) & VERY LOW \\
Quality of life & MD 0.65 lower (4.79 lower to 3.49 higher) & 36 (1 RCT) & VERY LOW \\
\hline
\end{tabular}

GRADE: Grading of Recommendations, Assessment, Development and Evaluation, ADL: activity of daily living, SMD: standardized mean difference, SD: standard deviation, BPSD: behavioral and psychological symptoms of dementia

were not statistically significant, other therapies also improved ADL. In previous systematic reviews on the effect of NPI on ADL of people with dementia of any severity, exercise therapy and light therapy were beneficial in improving ADL. ${ }^{14,23}$ Since the study on light therapy was weighted as low as $8 \%$ in the meta-analysis, we cannot conclude the differential efficacy of NPI on ADL by intervention type (Table 2).
In the current study, NPI were not effective in reducing the overall BPSD, agitation, or anxiety. However, in the subgroup analyses, music therapy was effective in reducing the overall BPSD with a medium effect size. Two previous meta-analyses reported small to moderate effects of music therapy on BPSD in people with dementia of any severity, ${ }^{17,22}$ while one meta-analysis by Chang et $\mathrm{al}^{26}$ reported no benefits of music 
therapy on BPSD in PWMSD. ${ }^{26}$ However, 4 out of 6 studies included in their meta-analysis did not meet the inclusion criteria for the current meta-analysis; one was not a $\mathrm{RCT}^{73}$ one did not use formal criteria to diagnose dementia, ${ }^{74,75}$ and the other included mild dementia in the analysis. ${ }^{76}$

Although NPI had a medium-size effect on depression in PWMSD, this result should be interpreted with caution due to high heterogeneity and low certainty of the evidence. The subgroup analyses showed that massage therapy, though weighted only $18.1 \%$, was effective in reducing depression in PWMSD. Previous systematic reviews on the effects of NPI on depression in people with dementia of any severity ${ }^{15,16,77-80}$ also showed that massage therapy was effective in reducing depression.

NPI were not shown to be beneficial to cognitive function of PWMSD; this is consistent with the findings of previous systematic reviews. ${ }^{15-17,23}$

To the best of our knowledge, this is the first systematic review and meta-analysis to examine the efficacy of NPI only in PWMSD. However, the current study had several limitations. First, the certainty of evidence drawn from the current study was medium to very low, and publication bias could not be tested due to the limited number of studies included in the meta-analyses. We included the studies that diagnosed dementia using formal diagnostic criteria and provided the severity of dementia using validated measures in the current meta-analysis. Many studies did not specify the diagnostic criteria for dementia and/or the severity of dementia. Second, we employed fixed-effect models ${ }^{29}$ for the following reasons: 1) the number of studies integrated into the analysis was too small to estimate the between-study variance, 2) study populations included in the current study were more homogenous than those included in previous meta-analyses, because we included populations with moderate to severe dementia only, and 3) the aim of the current study was to examine the overall effect of NPI. However, the heterogeneity between studies was still high in the current analysis.

\section{CONCLUSION}

Although the certainty of evidence was moderate or low, the current systematic review found that NPI had a beneficial effect on ADL and depression in PWMSD.

\section{Acknowledgments}

This systematic review was supported by a grant from the National Institute of Dementia of Korea [grant no. NIDR-1703-0018], and the Korean Health Technology R\&D Project, Ministry of Health and Welfare, Republic of Korea [grant no. HI09C1379 (A092077)]. Also, this work was supported by KyungPook National University IACT grant funded by the Korea government (MSIT) (Humancare Contents Dvelopment).

\section{Conflicts of Interest}

The authors have no potential conflicts of interest to disclose.

\section{Author Contributions}

$\mathrm{RN}, \mathrm{YJK}$, and KWK created the search strategy and performed the search. $\mathrm{RN}$, JY, and $\mathrm{YY}$ independently extracted relevant data from articles and assessed the methodological quality and RoB of the studies. YJK, SB, KWK and $\mathrm{KK}$ interpreted the data and judged included studies and theirs RoB. $\mathrm{RN}$ and KK drafted the manuscript, and KWK revised the manuscript content. KK supervised the study. All authors read and approved the final manuscript.

\section{ORCID iDs}

Ki Woong Kim https://orcid.org/0000-0002-1103-3858

Riyoung Na https://orcid.org/0000-0002-2272-9981

\section{REFERENCES}

1. Lyketsos CG, Steinberg M, Tschanz JT, Norton MC, Steffens DC, Breitner JC. Mental and behavioral disturbances in dementia: findings from the Cache County Study on Memory in Aging. Am J Psychiatry 2000; 157:708-714.

2. Campbell N, Ayub A, Boustani MA, Fox C, Farlow M, Maidment I, et al. Impact of cholinesterase inhibitors on behavioral and psychological symptoms of Alzheimer's disease: a meta-analysis. Clin Interv Aging 2008;3:719-728.

3. Ballard C, Margallo-Lana M, Juszczak E, Douglas S, Swann A, Thomas A, et al. Quetiapine and rivastigmine and cognitive decline in Alzheimer's disease: randomised double blind placebo controlled trial. BMJ 2005;330:874.

4. Sink KM, Holden KF, Yaffe K. Pharmacological treatment of neuropsychiatric symptoms of dementia: a review of the evidence. JAMA 2005; 293:596-608.

5. Buckley JS, Salpeter SR. A risk-benefit assessment of dementia medications: systematic review of the evidence. Drugs Aging 2015;32:453-467.

6. Lao K, Ji N, Zhang X, Qiao W, Tang Z, Gou X. Drug development for Alzheimer's disease: review. J Drug Target 2018:1-10.

7. Fraser LA, Liu K, Naylor KL, Hwang YJ, Dixon SN, Shariff SZ, et al. Falls and fractures with atypical antipsychotic medication use: a population-based cohort study. JAMA Intern Med 2015;175:450-452.

8. Wang PS, Schneeweiss S, Avorn J, Fischer MA, Mogun H, Solomon $\mathrm{DH}$, et al. Risk of death in elderly users of conventional vs. atypical antipsychotic medications. N Engl J Med 2005;353:2335-2341.

9. Cohen-Mansfield J. Non-pharmacological interventions for persons with dementia: what are they and how should they be studied? Int Psychogeriatr 2018;30:281-283.

10. Sorbi S, Hort J, Erkinjuntti T, Fladby T, Gainotti G, Gurvit H, et al. EFNS-ENS Guidelines on the diagnosis and management of disorders associated with dementia. Eur J Neurol 2012;19:1159-1179.

11. Laver K, Cumming RG, Dyer SM, Agar MR, Anstey KJ, Beattie E, et al. Clinical practice guidelines for dementia in Australia. Med J Aust 2016; 204:191-193.

12. Abraha I, Rimland JM, Trotta FM, Dell'Aquila G, Cruz-Jentoft A, Petrovic $M$, et al. Systematic review of systematic reviews of non-pharmacological interventions to treat behavioural disturbances in older patients with dementia. The SENATOR-OnTop series. BMJ Open 2017;7: e012759.

13. Dyer SM, Harrison SL, Laver K, Whitehead C, Crotty M. An overview of systematic reviews of pharmacological and non-pharmacological interventions for the treatment of behavioral and psychological symptoms of dementia. Int Psychogeriatr 2018;30:295-309.

14. McDermott O, Charlesworth G, Hogervorst E, Stoner C, Moniz-Cook E, Spector A, et al. Psychosocial interventions for people with dementia: a synthesis of systematic reviews. Aging Ment Health 2018 [Epub ahead of print]. 
15. Tsoi KKF, Chan JYC, Ng YM, Lee MMY, Kwok TCY, Wong SYS. Receptive music therapy is more effective than interactive music therapy to relieve behavioral and psychological symptoms of dementia: a systematic review and meta-analysis. J Am Med Dir Assoc 2018;19:568-576. e563.

16. Orgeta V, Qazi A, Spector A, Orrell M. Psychological treatments for depression and anxiety in dementia and mild cognitive impairment: systematic review and meta-analysis. Br J Psychiatry 2015;207:293-298.

17. van der Steen JT, Smaling HJA, van der Wouden JC, Bruinsma MS, Scholten R, Vink AC. Music-based therapeutic interventions for people with dementia. Cochrane Database Syst Rev 2018;7:CD003477.

18. Laver K, Dyer S, Whitehead C, Clemson L, Crotty M. Interventions to delay functional decline in people with dementia: a systematic review of systematic reviews. BMJ Open 2016;6:e010767.

19. Brasure M, Jutkowitz E, Fuchs E, Nelson VA, Kane RA, Shippee T, et al. Nonpharmacologic Interventions for Agitation and Aggression in Dementia. Comparative Effectiveness Review No. 177. Minnesota: AHRQ Publication; 2016.

20. Brodaty H, Arasaratnam C. Meta-analysis of nonpharmacological interventions for neuropsychiatric symptoms of dementia. Am J Psychiatry 2012;169:946-953.

21. Burns A, Guthrie E, Marino-Francis F, Busby C, Morris J, Russell E, et al. Brief psychotherapy in Alzheimer's disease: Randomised controlled trial. Br J Psychiatry 2005;187:143-147.

22. Ueda T, Suzukamo Y, Sato M, Izumi SI. Effects of music therapy on behavioral and psychological symptoms of dementia: a systematic review and meta-analysis. Ageing Res Rev 2013;12:628-641.

23. Forbes D, Blake CM, Thiessen EJ, Peacock S, Hawranik P. Light therapy for improving cognition, activities of daily living, sleep, challenging behaviour, and psychiatric disturbances in dementia. Cochrane Database Syst Rev 2014;(2):CD003946.

24. Kverno KS, Black BS, Nolan MT, Rabins PV. Research on treating neuropsychiatric symptoms of advanced dementia with non-pharmacological strategies, 1998-2008: a systematic literature review. Int Psychogeriatr 2009;21:825-843.

25. Boote J, Lewin V, Beverley C, Bates J. Psychosocial interventions for people with moderate to severe dementia: a systematic review. Clin Effect Nurs 2006;9:e1-e15.

26. Chang YS, Chu H, Yang CY, Tsai JC, Chung MH, Liao YM, et al. The efficacy of music therapy for people with dementia: a meta-analysis of randomised controlled trials. J Clin Nurs 2015;24:3425-3440.

27. Pedersen SKA, Andersen PN, Lugo RG, Andreassen M, Sütterlin S. Effects of music on agitation in dementia: a meta-analysis. Front Psychol 2017;8:742.

28. Shamseer L, Moher D, Clarke M, Ghersi D, Liberati A, Petticrew M, et al. Preferred reporting items for systematic review and meta-analysis protocols (PRISMA-P) 2015: elaboration and explanation. BMJ 2015;349: g7647.

29. Higgins J, Green S. Cochrane Handbook for Systematic Reviews of Interventions Version 5.1.0 [updated March 2011]. The Cochrane Collaboration 2011. Available from http://handbook.cochrane.org.

30. Na R, Kim YJ, Kim K, Kim KW. A systematic review of nonpharmacological interventions for moderate to severe dementia: A study protocol for a systematic review and meta-analysis. Psychiatry Investig 2018;15: 417-423.

31. American Psychiatric Association. DSM-IV-TR: Diagnostic and Statistical Manual of Mental Disorders, Text Revision. Washington, DC: American Psychiatric Association Publishing; 2000.

32. Association AP. Diagnostic and Statistical Manual of Mental Health Disorders (DSM-III-R). Washington DC: American Psychiatric Association; 1987.

33. Association AP. Diagnostic and statistical manual of mental disorders $\left(\right.$ DSM- $\left.5^{\circ}\right)$. Washington DC: American Psychiatric Pub; 2013.

34. World Health Organization (1992). The ICD-10 classification of mental and behavioural disorders: clinical descriptions and diagnostic guide- lines. World Health Organization. http://www.who.int/iris/handle/ $10665 / 37958$

35. McKhann GM, Knopman DS, Chertkow H, Hyman BT, Jack CR, Kawas $\mathrm{CH}$, et al. The diagnosis of dementia due to Alzheimer's disease: Recommendations from the National Institute on Aging-Alzheimer's Association workgroups on diagnostic guidelines for Alzheimer's disease. Alzheimers Dement 2011;7:263-269.

36. McKhann G, Drachman D, Folstein M, Katzman R, Price D, Stadlan EM. Clinical diagnosis of Alzheimer's disease Report of the NINCDSADRDA Work Group ${ }^{*}$ under the auspices of Department of Health and Human Services Task Force on Alzheimer's Disease. Neurology 1984; 34:939-944.

37. Morris JC. Clinical dementia rating: a reliable and valid diagnostic and staging measure for dementia of the Alzheimer type. Int Psychogeriatr 1997;9:173-176.

38. Reisberg B, Ferris SH, de Leon MJ, Crook T. The Global Deterioration Scale for assessment of primary degenerative dementia. Am J Psychiatry 1982;139:1136-1139.

39. Sclan SG, Reisberg B. Functional assessment staging (FAST) in Alzheimer's disease: reliability, validity, and ordinality. Int Psychogeriatr 1992; 4: 55-69.

40. Folstein MF, Robins LN, Helzer JE. The mini-mental state examination. Arch Gen Psychiatry 1983;40:812.

41. Rabins PV, Blacker D, Rovner BW, Rummans T, Schneider LS, Tariot PN, et al. American Psychiatric Association practice guideline for the treatment of patients with Alzheimer's disease and other dementias. Second edition. Am J Psychiatry 2007;164(12 Suppl):5-56.

42. Seo HJ, Kim SY, Lee YJ, Jang BH, Park JE, Sheen SS, et al. A newly developed tool for classifying study designs in systematic reviews of interventions and exposures showed substantial reliability and validity. J Clin Epidemiol 2016;70:200-205.

43. Higgins JP, Altman DG, Gøtzsche PC, Jüni P, Moher D, Oxman AD, et al. The Cochrane Collaboration's tool for assessing risk of bias in randomised trials. BMJ 2011;343:d5928.

44. Guyatt G, Oxman AD, Akl EA, Kunz R, Vist G, Brozek J, et al. GRADE guidelines: 1. Introduct ion-GRADE evidence profiles and summary of findings tables. J Clin Epidemiol 2011;64:383-394.

45. Rolland Y, Pillard F, Klapouszczak A, Reynish E, Thomas D, Andrieu S, et al. Exercise program for nursing home residents with Alzheimer's disease: A 1-year randomized, controlled trial. J Am Geriatr Soc 2007;55: 158-165.

46. Burns A, Allen H, Tomenson B, Duignan D, Byrne J. Bright light therapy for agitation in dementia: a randomized controlled trial. Int Psychogeriatr 2009;21:711-721.

47. Woods DL, Beck C, Sinha K. The effect of therapeutic touch on behavioral symptoms and cortisol in persons with dementia. Forsch Komplementmed 2009;16:181-189.

48. Raglio A, Bellelli G, Traficante D, Gianotti M, Ubezio MC, Gentile S, et al. Efficacy of music therapy treatment based on cycles of sessions: a randomised controlled trial. Aging Ment Health 2010;14:900-904.

49. Roach KE, Tappen RM, Kirk-Sanchez N, Williams CL, Loewenstein D. A randomized controlled trial of an activity specific exercise program for individuals with Alzheimer disease in long-term care settings. J Geriatr Phys Ther 2011;34:50-56.

50. Sakamoto M, Ando H, Tsutou A. Comparing the effects of different individualized music interventions for elderly individuals with severe dementia. Int Psychogeriatr 2013;25:775-784.

51. Hutson C, Orrell M, Dugmore O, Spector A. Sonas: a pilot study investigating the effectiveness of an intervention for people with moderate to severe dementia. Am J Alzheimers Dis Other Demen 2014;29:696-703.

52. Narme P, Clément S, Ehrlé N, Schiaratura L, Vachez S, Courtaigne B, et al. Efficacy of musical interventions in dementia: evidence from a randomized controlled trial. J Alzheimers Dis 2014;38:359-369.

53. Bürge E, Berchtold A, Maupetit C, Bourquin NM-P, von Gunten A, Ducraux D, et al. Does physical exercise improve ADL capacities in people 
over 65 years with moderate or severe dementia hospitalized in an acute psychiatric setting? A multisite randomized clinical trial. Int Psychogeriatr 2017;29:323-332.

54. Rodríguez-Mansilla J, González López-Arza MV, Varela-Donoso E, Montanero-Fernández J, González Sánchez B, Garrido-Ardila EM. The effects of ear acupressure, massage therapy and no therapy on symptoms of dementia: a randomized controlled trial. Clin Rehabil 2015;29:683693.

55. Toots A, Littbrand H, Lindelöf N, Wiklund R, Holmberg H, Nordström P, et al. Effects of a High-Intensity Functional Exercise Program on Dependence in Activities of Daily Living and Balance in Older Adults with Dementia. J Am Geriatr Soc 2016;64:55-64.

56. Katz S, Ford AB, Moskowitz RW, Jackson BA, Jaffe MW. Studies of illness in the aged: The index of adl: a standardized measure of biological and psychosocial function. JAMA 1963;185:914-919.

57. Mahoney FI, Barthel D. Functional evaluation: the Barthel Index. Md State Med J 1965;14:56-61.

58. Collin C, Wade DT, Davies S, Horne V. The Barthel ADL Index: A reliability study. Int Disabil Stud 1988;10:61-63.

59. Robinson R. Some problems of clinical trials in elderly people. Gerontol Clin (Basel) 1961;3:247-257.

60. Cummings JL, Mega M, Gray K, Rosenberg-Thompson S, Carusi DA, Gornbein J. The Neuropsychiatric Inventory comprehensive assessment of psychopathology in dementia. Neurology 1994;44:2308-2314.

61. Cummings JL. The Neuropsychiatric Inventory Assessing psychopathology in dementia patients. Neurology 1997;48:10S-16S.

62. Kaufer DI, Cummings JL, Ketchel P, Smith V, MacMillan A, Shelley T, et al. Validation of the NPI-Q, a brief clinical form of the Neuropsychiatric Inventory. J Neuropsychiatry Clin Neurosci 2000;12:233-239.

63. Reisberg B, Auer SR, Monteiro IM. Behavioral pathology in Alzheimer's disease (BEHAVE-AD) rating scale. Int Psychogeriatr 1997;8:301308 .

64. Allen N, Gordon S, Hope T, Burns A. Manchester and Oxford Universities scale for the psychopathological assessment of dementia (MOUSEPAD). Br J Psychiatry 1996;169:293-307.

65. Kørner A, Lauritzen L, Abelskov K, Gulmann N, Marie Brodersen A, Wedervang-Jensen $\mathrm{T}$, et al. The geriatric depression scale and the cornell scale for depression in dementia. A validity study. Nord J Psychiatry 2006; 60:360-364.

66. Leentjens AF, Verhey FR, Lousberg R, Spitsbergen H, Wilmink FW. The validity of the Hamilton and Montgomery-Åsberg depression rating scales as screening and diagnostic tools for depression in Parkinsons disease. Int J Geriatr psychiatry 2000;15:644-649.
67. Müller-Thomsen T, Arlt S, Mann U, Maß R, Ganzer S. Detecting depression in Alzheimer's disease: evaluation of four different scales. Arch Clin Neuropsychol 2005;20:271-276.

68. Cohen-Mansfield J, Marx MS, Rosenthal AS. A description of agitation in a nursing home. J Gerontol 1989;44:M77-M84.

69. Bliwise DL, Carroll JS, Lee KA, Nekich JC, Dement WC. Sleep and "sundowning" in nursing home patients with dementia. Psychiatry Res 1993; 48:277-292.

70. Shankar K, Walker M, Frost D, Orrell M. The development of a valid and reliable scale for rating anxiety in dementia (RAID). Aging Ment Health 1999;3:39-49.

71. Spielberger CD, Gorsuch RL, Lushene R, Vagg PR, Jacobs GA. Manual for the State-Trait Anxiety Inventory. Palo Alto, CA: Consulting Psychologists Press; 1983.

72. Tariot PN, Farlow MR, Grossberg GT, Graham SM, McDonald S, Gergel I, et al. Memantine treatment in patients with moderate to severe Alzheimer disease already receiving donepezil: a randomized controlled trial. JAMA 2004;291:317-324.

73. Raglio A, Bellelli G, Traficante D, Gianotti M, Ubezio MC, Villani D, et al. Efficacy of music therapy in the treatment of behavioral and psychiatric symptoms of dementia. Alzheimer Dis Assoc Disord 2008;22: 158162.

74. Janata P, Tomic ST, Haberman JM. Sensorimotor coupling in music and the psychology of the groove. J Exp Psychol Gen 2012;141:54-75.

75. Sung HC, Chang AM, Abbey J. The effects of preferred music on agitation of older people with dementia in Taiwan. Int J Geriatr Psychiatry 2006;21:999-1000.

76. Lin Y, Chu H, Yang CY, Chen CH, Chen SG, Chang HJ, et al. Effectiveness of group music intervention against agitated behavior in elderly persons with dementia. Int J Geriatr Psychiatry 2011;26:670-678.

77. Scales K, Zimmerman S, Miller SJ. Evidence-based nonpharmacological practices to address behavioral and psychological symptoms of dementia. Gerontologist 2018;58(suppl 1):S88-S102.

78. Wu J, Wang Y, Wang Z. The effectiveness of massage and touch on behavioural and psychological symptoms of dementia: a quantitative systematic review and meta-analysis. J Adv Nurs 2017; 73: 2283-2295.

79. Zhang Y, Cai J, An L, Hui F, Ren T, Ma H, et al. Does music therapy enhance behavioral and cognitive function in elderly dementia patients? A systematic review and meta-analysis. Ageing Res Rev 2017;35:1-11.

80. Folkerts AK, Roheger M, Franklin J, Middelstädt J, Kalbe E. Cognitive interventions in patients with dementia living in long-term care facilities: Systematic review and meta-analysis. Arch Gerontol Geriatr 2017; 73:204-221. 